\title{
INNER-SPHERE AND OUTER-SPHERE COORDINATION OF WATER IN AQUATRIPHENYLTIN BIS(N,N-DIMETHYLDITHIOCARBAMYL)ACETATE HYDRATE
}

\author{
Seik Weng $\mathrm{Ng}^{1}$ and V.G. Kumar Das ${ }^{2}$ \\ ${ }^{1}$ Institute of Advanced Studies \\ 2 Department of Chemistry, University of Malaya, 59100 Kuala Lumpur, Malaysia
}

\begin{abstract}
Aquatriphenyltin $\quad \operatorname{bis}(N, N$-dimethyldithiocarbamyl)acetate hydrate crystallizes as zig-zag chains that are hydrogen-bonded to the lattice water molecules to give rise to a tight network structure. The structure is the first example of a hydrated organotin compound in which water participates in both inner- and outer-sphere coordination to tin.
\end{abstract}

\section{Introduction}

X-ray diffraction studies on hydrated organotin compounds have provided several examples of water engaged in either inner-sphere or outer-sphere coordination to tin [1-3]. For inner-sphere coordinated compounds, accumulated evidence suggests that these compounds tend to pack in arrangements in which the number of hydrogen bonding interactions involving the coordinated water molecule is maximized. For example, the common carboxyl bridging mode of triorganotin carboxylates is relinquished in favor of such interactions in the corresponding hydrated carboxylates, the water molecule binding to the carbonyl oxygen atom and to other Lewis-basic atoms of the carboxylato anion in aquatriphenyltin 8-quinolyloxyacetate [2].

Triorganotin carboxylates are generally synthesized by treating a triorganotin hydroxide or hemioxide with the carboxylic acid in an alcoholic or hydrocarbon medium, as exemplified by the reaction of triphenyltin hydroxide and $\mathrm{N}, \mathrm{N}$-dimethyldithiocarbamylacetic acid [4,5]. For the coresponding reaction of triphenyltin hydroxide with the homologous $\operatorname{bis}(N, N$-dimethyldithiocarbamyl)acetic acid, the choice of solvent medium 
appeared to influence the product: the use of ethanol afforded a $1 / 1$ ethanol adduct of the expected ester [4], whereas the use of toluene yielded the hydrated compound, whose crystal structure is described here.

\section{Experimental}

The compound was synthesized by condensing equimolar amounts of $\operatorname{bis}(N, N$-dimethyldithiocarbamyl)acetic acid and triphenyltin hydroxide in a small volume of boiling toluene. The filtered solution when cooled slowly afforded tan-colored crystals of the title compound. An irregularly-shaped crystal, $0.43 \mathrm{x}$ $0.43 \times 0.43 \mathrm{~mm}$, was used for diffraction analysis. Unit-cell constants were refined from the 25 strongest reflections in the $14^{\circ} \leq \Theta \leq 15^{\circ}$ shell. The 5724 reflections were measured by $\omega-2 \Theta$ scans up to $2 \Theta=50^{\circ}$ (collection range: $-16 \leq$ $h<0,-18<k<0,-17<l<17$ ), and were corrected for a decay of $6.2 \%$ over 33.9 $\mathrm{h}$ exposure to X-rays (av. corr. $=1.0325$ ) and for absorption [6] (transmission factors $=89.18-99.97 \%)$. After removing systematically absent reflections, the 4395 of the 5246 independent reflections $\left(R_{\mathrm{int}}=0.016\right)$ satisfying the $I>3 \sigma(I)$ criterion were used for solution and refinement. The structure was solved by direct phase determination; non- $\mathrm{H}$ atoms were refined anisotropically; the aromatic $\mathrm{H}$-atoms were placed at calculated positions $\left(\mathrm{C}-\mathrm{H}=0.95 \AA, B=5 \AA^{2}\right)$ and allowed to ride on their parent $\mathrm{C}$-atoms. The $\mathrm{H}$-atoms of the two water molecules were located and refined $\left(B=5 \AA^{2}\right)$. Refinement on $F$ for 346 variables converged with a shift-to-error ratio of less than 0.01 to $R=0.034 ; R_{\mathrm{w}}=0.042$ and $S=0.900$ for $w=\left[\sigma(F)^{2}+(0.02 F)^{2}+1\right]^{-1}[7]$. The final difference map had peaks between $-0.17(3)$ and 1.13(3) $e \AA^{-3}$; the 1.13(3) $e \AA^{-3}$ peak was located between the S4 and C20 atoms. Computations with performed with the MolEN package on a MicroVAX minicomputer [8]. The atomic labeling is shown in Fig. 1.

Crystal data: $\mathrm{C}_{26} \mathrm{H}_{32} \mathrm{~N}_{2} \mathrm{O}_{4} \mathrm{~S}_{4} \mathrm{Sn}, \quad F W=683.50$, monoclinic, $P 2_{1} / a$ (non-standard No. 14), $a=14.414(1), b=13.7824(6), c=15.567(1) \AA, \beta=$ $105.143(4)^{\circ}, V=2985.1(4) \AA^{3}, D=1.521 \mathrm{~g} \mathrm{~cm}^{-3}, F(000)=1392, \mu=11.59 \mathrm{~cm}^{-1}$ for $Z=4$.

\section{Results and discussion}

Aquatriphenyltin $\operatorname{bis}(N ; N$-dimethyldithiocarbamyl)acetate hydrate crystallizes as a five-coordinate molecule (Fig. 1) whose geometry at tin is a trigonal bipyramid having equatorial orientation of the phenyl groups $(\Sigma \mathrm{C}-\mathrm{Sn}-\mathrm{C}$ $\left.=358.7(6)^{\circ}\right)$. The axial positions are occupied by the coordinated water $(\mathrm{Sn}-\mathrm{O}=$ $2.388(3) \AA)$ and the esteryl oxygen $(\mathrm{Sn}-\mathrm{O}=2.160(3) \AA)$ atoms, which subtend an 
Table 1. Atomic coordinates and equivalent ${ }^{a}$ isotropic temperature factors

\begin{tabular}{lclll}
\hline Atom & $x$ & $y$ & $z$ & $B_{\text {eq }}\left(\AA^{2}\right)$ \\
& & & & \\
Sn1 & $0.10353(2)$ & $0.37076(2)$ & $0.76530(2)$ & $2.528(5)$ \\
S1 & $0.33489(8)$ & $0.61642(8)$ & $0.71109(7)$ & $3.47(2)$ \\
S2 & $0.2784(1)$ & $0.51930(9)$ & $0.53299(8)$ & $4.64(3)$ \\
S3 & $0.46558(8)$ & $0.4984(1)$ & $0.83861(7)$ & $3.92(2)$ \\
S4 & $0.58856(9)$ & $0.5367(1)$ & $0.71233(9)$ & $4.64(3)$ \\
O1 & $0.2365(2)$ & $0.4420(2)$ & $0.7635(2)$ & $3.45(6)$ \\
O2 & $0.3258(2)$ & $0.3316(2)$ & $0.7183(2)$ & $3.73(6)$ \\
Ow1 & $-0.0551(2)$ & $0.3247(2)$ & $0.7692(2)$ & $3.72(7)$ \\
Ow2 & $0.1935(3)$ & $0.5429(4)$ & $0.3091(3)$ & $7.1(1)$ \\
N1 & $0.2495(3)$ & $0.7055(3)$ & $0.5643(2)$ & $3.77(8)$ \\
N2 & $0.6383(3)$ & $0.5702(3)$ & $0.8856(3)$ & $4.46(9)$ \\
C1 & $0.1582(3)$ & $0.2686(3)$ & $0.8687(3)$ & $3.01(8)$ \\
C2 & $0.1102(4)$ & $0.2440(4)$ & $0.9319(3)$ & $4.1(1)$ \\
C3 & $0.1512(4)$ & $0.1827(4)$ & $1.0017(3)$ & $5.0(1)$ \\
C4 & $0.2402(4)$ & $0.1440(4)$ & $1.0088(4)$ & $5.4(1)$ \\
C5 & $0.2866(4)$ & $0.1639(4)$ & $0.9457(4)$ & $5.7(1)$ \\
C6 & $0.2471(3)$ & $0.2261(4)$ & $0.8763(3)$ & $4.5(1)$ \\
C7 & $0.0586(3)$ & $0.5091(3)$ & $0.8009(3)$ & $2.89(8)$ \\
C8 & $0.0087(3)$ & $0.5182(4)$ & $0.8656(3)$ & $3.80(9)$ \\
C9 & $-0.0192(3)$ & $0.6088(4)$ & $0.8878(3)$ & $4.7(1)$ \\
C10 & $0.0004(4)$ & $0.6904(4)$ & $0.8460(4)$ & $5.0(1)$ \\
C11 & $0.0503(4)$ & $0.6821(4)$ & $0.7832(4)$ & $5.0(1)$ \\
C12 & $0.0804(3)$ & $0.5928(3)$ & $0.7609(3)$ & $3.7(1)$ \\
C13 & $0.0705(3)$ & $0.3172(3)$ & $0.6335(3)$ & $2.87(8)$ \\
C14 & $0.0650(3)$ & $0.3771(4)$ & $0.5606(3)$ & $4.3(1)$ \\
C15 & $0.0426(4)$ & $0.3385(5)$ & $0.4760(3)$ & $5.7(1)$ \\
C16 & $0.0254(4)$ & $0.2407(5)$ & $0.4633(3)$ & $5.8(1)$ \\
C17 & $0.0306(4)$ & $0.1820(4)$ & $0.5334(4)$ & $5.4(1)$ \\
C18 & $0.0534(3)$ & $0.2196(4)$ & $0.6192(3)$ & $4.2(1)$ \\
C19 & $0.3080(3)$ & $0.4142(3)$ & $0.7364(3)$ & $2.66(8)$ \\
C20 & $0.3809(3)$ & $0.4945(3)$ & $0.7306(3)$ & $2.97(8)$ \\
C21 & $0.2824(3)$ & $0.6188(3)$ & $0.5947(3)$ & $3.25(8)$ \\
C22 & $0.2537(4)$ & $0.7889(4)$ & $0.6215(4)$ & $5.5(1)$ \\
C23 & $0.2086(4)$ & $0.7209(4)$ & $0.4696(3)$ & $5.0(1)$
\end{tabular}


C24

$0.5732(3)$

$0.5391(3)$

$0.8141(3)$

3.24(9)

C25

$0.6202(4)$

$0.5796(5)$

$0.9731(3)$

$5.8(1)$

C26

$0.7332(4)$

$0.5990(5)$

$0.8790(5)$

$6.8(2)$

${ }^{a} B_{\mathrm{eq}}=4 / 3\left[a^{2} B(1,1)+b^{2} B(2,2)+c^{2} B(3,3)+a b(\cos \gamma) B(1,2)+a c(\cos \beta) B(1,3)+\right.$ $b c(\cos \alpha) B(2,3)]$

angle of $168.4(1)^{\circ}$ at tin. The dative bond distance is similar to distances found found in the two independent molecules of aquatriphenyltin 8-quinolyloxyacetate [2]. The covalent bond distance, on the other hand, is much shorter than those [9] found in most carboxylate-bridged triphenyltin alkanoates, but are comparable with those [10] documented in four-coordinate triphenyltin arylcarboxylates.

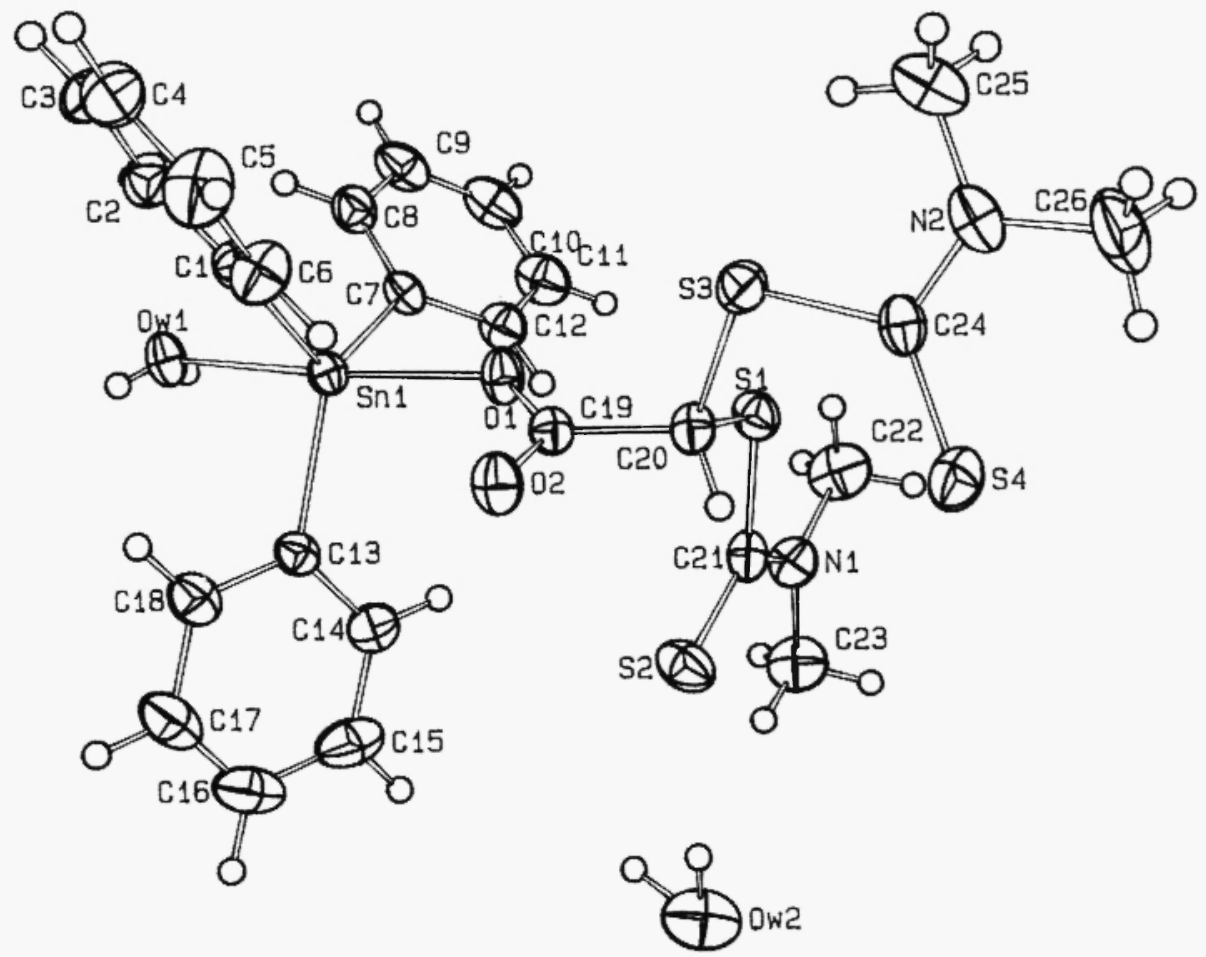

Fig. 1. Geometry of the tin atom in aquatriphenyltin bis $(N, N$-dimethyldithiocarbamyl)acetate hydrate. Selected bond distances and angles: $\mathrm{Snl}-\mathrm{O} 1=2.160(3), \mathrm{Snl}-\mathrm{O} w 1$ $=2.388(3), \mathrm{Sn} 1-\mathrm{C} 1=2.129(4), \mathrm{Sn} 1-\mathrm{C} 7=2.133(4), \mathrm{Sn} 1-\mathrm{C} 13=2.114(4) \AA \AA$; $1-\mathrm{Sn} 1-\mathrm{O} w 1$ $=168.4(1), \mathrm{O} 1-\mathrm{Sn} 1-\mathrm{Cl}=99.0(1), \mathrm{O} 1-\mathrm{Sn} 1-\mathrm{C} 7=85.9(1), \mathrm{O} 1-\mathrm{Sn} 1-\mathrm{C} 13=97.0(1)$, Ow1-Sn1-C1 = 88.4(1), Ow1-Sn1-C7 = 82.7(1), Ow1-Sn1-C13 = 87.6(1), C1-Sn1-C7 = 118.2(2), C1-Sn1-C13 = 116.6(2), C7-Sn1-C13 = 123.9(2) . 
The aquatriphenyltin bis( $N, N$-dimethyldithiocarbamyl)acetate molecules are linked by hydrogen bonds involving the coordinated water and the free carbonyl oxygen atom of the glide-related carboxylato moiety $(\mathrm{O} \cdots=2.740(4) \AA)$ into a zig-zag chain along the $a$-axis. The chains are in turn linked to each other through the lattice water molecule, which hydrogen bonds to the thione sulfur atoms belonging to two carboxylato anions $(\mathrm{O} \cdots \mathrm{S}=3.394(5), 3.426(5) \AA)$, and to the coordinated water molecule $(\mathrm{O} \mathrm{O}=2.743(6) \AA)$ (Fig. 2$)$ to give rise to a three-dimensional network structure. The structure is an unusual example of a hydrated organotin compound in which water is found in both the inner- and outer-coordination sphere of tin. A similar dual coordination mode for ethanol in bis(triphenyltin) succinate.2ethanol has been recently identified [11].

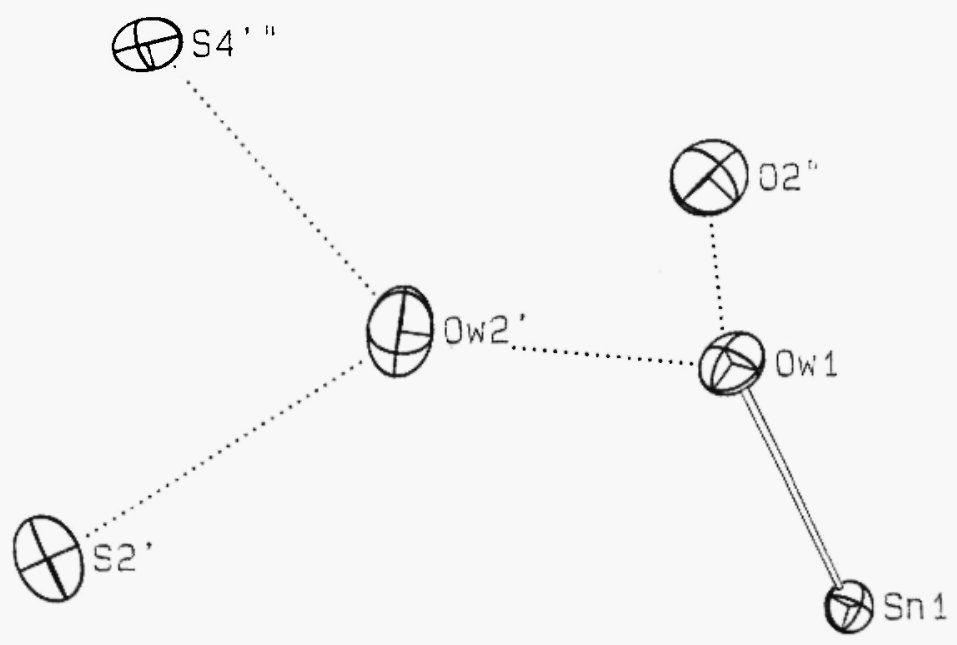

Fig. 2. Coordination of water to tin. Bond distances and angles: $\mathrm{S} 2 \cdots \mathrm{O} w 2=3.394(5)$, $\mathrm{S} 44^{\prime \prime \cdots} \mathrm{O} w 2^{\prime}=3.426(5), \mathrm{O} 2^{\prime \prime \prime} \mathrm{O} w 1=2.740(4), \mathrm{O} w 1^{\prime \prime} \mathrm{O} w 2^{\prime}=2.743(6) \AA ; \mathrm{Sn} 1-\mathrm{O} w 1{ }^{\prime \prime O} 2^{\prime \prime}=$

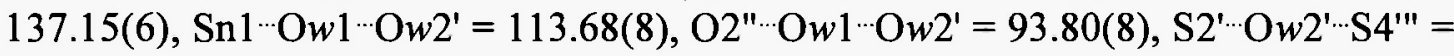

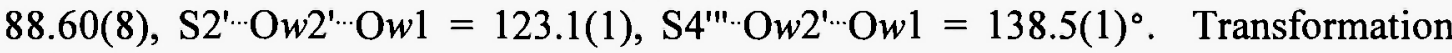
codes: (') $-x, 1-y, 1-z$; (") $x-1 / 2 \frac{1}{2}-y, z$; ("') $x-1, y, z$.

\section{Acknowledgments}

This work was generously supported by the National Science Council for R \& D (Grant No. 2-07-04-06). 


\section{References}

1 E.J. Gabe, F.L. Lee and F.E. Smith, Inorg. Chim. Acta, 90 (1984) L11.

2 V.G. Kumar Das, W. Chen, S.W. Ng and T.C.W. Mak, J. Organomet. Chem., 322 (1987) 33.

3 F. Huber, H. Preut, E. Hoffmann and M. Gielen, Acta Crystallogr., C45 (1989) 51.

$4 \quad$ S.W. Ng and V.G. Kumar Das, J. Organomet. Chem., 409 (1991) 143.

5 S.W. Ng and V.G. Kumar Das, Acta Crystallogr., C51 (1995) 000.

6 A.C.T. North, D.C. Philips and F.S. Mathews, Acta Crystallogr., A24 (1968) 351.

7 R.C.G. Killean and J.L. Lawrence, Acta Crystallogr., B25 (1969) 1750.

8 Delft Instruments. MolEN Structure Determination System, Delft Instruments, X-ray Diffraction B.V., Röntgenweg 1, 2624 BD Delft, The Netherlands (1990).

9 S.W. Ng, W. Chen and V.G. Kumar Das, J. Organomet. Chem.. 345 (1988) 59.

10 S.W. Ng, V.G. Kumar Das, F. van Meurs, J.D. Schagen and L.H. Stravers, Acta Crystallogr., C45 (1989) 568.

11 S.W. Ng and V.G. Kumar Das, Malays. J. Sci., 15B (1994) 7.

Received: April 13, 1995 - Accepted: May 4, 1995 - Received revised camera-ready manuscript: June 20, 1995 